BRX TH-517

\title{
A Note on Matter Superenergy Tensors
}

\author{
S. Deser \\ Brandeis University \\ Department of Physics \\ Waltham, MA 02454, USA \\ E-mail: deser@brandeis.edu
}

\begin{abstract}
We consider Bel-Robinson-like higher derivative conserved two-index tensors $H_{\mu \nu}$ in simple matter models, following a recently suggested Maxwell field version. In flat space, we show that they are essentially equivalent to the true stress-tensors. In curved Ricci-flat backgrounds it is possible to redefine $H_{\mu \nu}$ so as to overcome non-commutativity of covariant derivatives, and maintain conservation, but they become model- and dimension- dependent, and generally lose their simple "BR" form.
\end{abstract}

Historically, the nonexistence of a local stress tensor in generally covariant theories such as Einstein's led to a successful search for the next-best thing, the covariantly conserved but fourindex and higher derivative Bel-Robinson (BR) tensor [1], quadratic in curvature. Being traceless in $\mathrm{D}=4$, it has no " $T_{\mu \nu}$-like" 2-index contraction. However, this discovery led ineluctably to an equally successful search [2] for matter analogs of BR, despite the presence of perfectly good $T_{\mu \nu}$ there. These quantities resemble BR in being of higher derivative order and quadratic in the "curvatures" of the corresponding fields. In particular, it has recently been shown [3] that there is a natural 2index conserved BR-version of the Maxwell tensor. In flat space QFT, operators $H_{\mu \nu}$ whose matrix elements behave like those of the stress-tensor are essentially proportional to it [4]: In momentum space, one expects a conserved symmetric 2-tensor to have the form $f\left(q^{2}\right) T_{\mu \nu}(q)$, up to (trivial) identically conserved terms. By continuity, one might expect some similar property to hold, at least for test fields, i.e., in Ricci-flat spaces. The results we obtain here confirm these expectations, at least for $H_{\mu \nu}$ of simple free field models. Using the simplest - scalar and vector - free field models we will first investigate their $H_{\mu \nu}$ in flat space and immediately verify the expectation that they (and their obvious generalizations) are indeed related to the corresponding $T_{\mu \nu}$ by form factors. In gravitational backgrounds we find that while $H_{\mu \nu}$ can be redefined to survive non-commutation of derivatives at least in Ricci-flat spaces, generically they lose their flat space attributes and become model- and dimension-dependent.

In flat space, the new tensors are respectively

$$
H_{\mu \nu}^{s} \equiv \phi_{\mu \alpha} \phi_{\nu}^{\alpha}-\frac{1}{2} \eta_{\mu \nu}\left(\phi_{\alpha \beta} \phi^{\alpha \beta}\right), \quad \phi_{\mu \alpha} \equiv \partial_{\alpha \mu}^{2} \phi
$$

and $[2]$

$$
H_{\mu \nu}^{v} \equiv F_{\mu \lambda \alpha} F_{\nu}^{\lambda \alpha}-\frac{1}{4} \eta_{\mu \nu}\left(F_{\lambda \beta \alpha} F^{\lambda \beta \alpha}\right), \quad F_{\mu \lambda \alpha} \equiv \partial_{\alpha} F_{\mu \lambda}
$$

for scalar and Maxwell fields, and exhibit the BR form, in terms of the "curvatures" $\phi_{\mu \nu}$ and $F_{\mu \lambda \alpha}$. [The extra derivatives on the fields are clearly completely transparent to taking divergences, 
symmetry, etc.] Our main point, however, is that, equally obviously, they are simply related to their corresponding stress tensors, through

$$
H_{\mu \nu}^{s}=\frac{1}{2} \square T_{\mu \nu}^{s}
$$

and

$$
H_{\mu \nu}^{v}=\frac{1}{2} \square T_{\mu \nu}^{\max } .
$$

Here and throughout we work entirely on-shell, since that is all that matters; in particular, we used the Maxwell field equation

$$
\square F_{\mu \nu}=0 .
$$

These examples, then, show that the $H_{\mu \nu}$ are precisely of the (momentum space) form $f\left(q^{2}\right) T_{\mu \nu}(q)$, devoid of independent content.

An excursion into curved space is less directly motivated, as there is no expected simple physical " $H-T$ " connection there. Still, it is mildly interesting that any results at all can be drawn in more general backgrounds. It is immediately clear that not only do the definitions $(1,2)$ of $H$ not imply $(3,4)$ but that in neither form does (covariant) conservation of $H$ follow from that of $T$. The reason is of course the non-commutativity among covariant derivatives, particularly $\left[D_{\mu}, \square\right] \neq 0$.

A resolution of this impasse exists, however, for spaces that are Ricci-flat: when $\square$ is replaced by the Lichnerowicz operator [5],

$$
\tilde{H}_{\mu \nu} \equiv(L T)_{\mu \nu} \equiv \square T_{\mu \nu}+2 R_{\mu \alpha \nu \beta} T^{\alpha \beta}
$$

commutativity is restored:

$$
D^{\mu}(L T)_{\mu \nu}=L\left(D^{\mu} T_{\mu \nu}\right)=0 .
$$

The curvature addition in (6) compensates for $\left[D^{\mu}, \square\right] \neq 0$. This happens in general for and only for Ricci-flat spaces, as is easily verified (recall that the divergence of the Riemann tensor is the curl of Ricci). We have thus rescued, albeit in restricted geometries, ${ }^{1}$ a conserved - redefined $\tilde{H}_{\mu \nu}$, but at the price of losing the desired BR form $(1,2)$. Can the (necessary) shift from $\square$ to $L$ be made compatible with $(1,2)$ ? The answer is a very qualified "yes", for $\mathrm{D}=4$ Maxwell only [3] and not otherwise. First we see why it does not work for scalars: the only difference between (3) and (1) is that the former contains extra terms $\sim \square D_{\mu} \phi$ (of course all derivative ordering must be carefully kept here!). But $\left[\square, D_{\mu}\right] \phi \sim D_{\mu} \square \phi+R_{\mu \alpha} \phi_{\alpha}$ and involves only the Ricci tensor, so we can drop it. This equality of (3) and (1), however, is also its drawback: since only $\tilde{H}=\square T+R T$ is conserved, it is not (1) alone, but the sum of (1) with $R T$ that is conserved. The Maxwell model, though only in $\mathrm{D}=4$, circumvents the above difficulty precisely because (4) differs from (2) by terms of the form $F(\square F)$ and these no longer vanish. Instead,

$$
0=D^{\alpha}\left[D_{\alpha} F_{\mu \nu}+D_{\nu} F_{\alpha \mu}+D_{\mu} F_{\mu \alpha}\right]=\square F_{\mu \nu}+\left\{R_{\nu \lambda \mu}^{\alpha} F_{\alpha}{ }^{\lambda}-(\nu \mu)\right\} .
$$

The ensuing $R F F$ terms in reducing (4) to (2), together with the $R T^{\max }$ part of $L T^{\max }$, cancel each other owing to a specifically $\mathrm{D}=4$ Weyl tensor identity [3]; the (covariantized) $H_{\mu \nu}^{v}$ of (2), being the reduction of $\tilde{H}_{\mu \nu}^{v}$, is conserved.

\footnotetext{
${ }^{1} \mathrm{In} \mathrm{D}=4, H_{\mu \nu}^{\max }$ is also conserved [3] on combined Einstein-Maxwell shell, i.e., for spaces whose Ricci tensor is proportional to $T_{\mu \nu}^{\max }$, due to the special properties of the Maxwell tensor in $\mathrm{D}=4$. This does not hold in other $\mathrm{D}$, nor even in $\mathrm{D}=4$, for the scalar model.
} 
Finally, a remark on the original, gravitational, BR tensor. In the present context, one might hope to construct it in terms of an underlying gauge-variant stress-tensor. This is impossible for a seemingly accidental reason: the trace of $B_{\mu \nu \lambda \beta}$ vanishes in $\mathrm{D}=4$ and so there is no $H_{\mu \nu}$-like candidate. [The only quadratic on-shell 2-index tensor is the square of Weyl, $C_{\mu \alpha \beta \gamma} C^{\nu \alpha \beta \gamma}$, which is a pure trace in $\mathrm{D}=4$.] More generally, this impossibility is understandable from the known fact [6] that (already in flat space) all $T_{\mu \nu}$ candidates for free linear spin $>1$ gauge fields vary by a superpotential term, under gauge transformations, one that cannot be removed by any curls or $\square$ operators: Hence $\square T_{\mu \nu}$ (or more general operators on $T_{\mu \nu}$ ) is still conserved but remains gaugevariant, even on shell. The required BR process here is more radical, taking individual "curls" of the factors in $T$, to reach the required $B \sim R R$ form.

In summary, we have seen that in flat space, ostensibly novel conserved symmetric BRlike extensions of matter $T_{\mu \nu}$ are really equivalent to it. In curved but Ricci-flat backgrounds, conservation of at least the extended $\tilde{H}_{\mu \nu}=(L T)_{\mu \nu}$, can be preserved. Even then (for all but the Maxwell field in $\mathrm{D}=4)$, since $(L T)$ is no longer of pure BR form (1) or (2), but acquires various non-minimal additions.

I acknowledge correspondence with the authors of [3]. This work was supported by NSF grant PHY99-73935.

\section{References}

[1] L. Bel, C.R. Acad. Sci., Paris 248, 1292 (1959); I. Robinson, unpublished lectures, Kings College, London (1958).

[2] M. Chevreton, Nuovo Cimento 34, 90 (1964).

[3] G. Bergqvist, I. Eriksson and J.M.M. Senovilla, gr-qc/0303046.

[4] See for example C.A. Orzalesi, J. Sucher and C.H. Woo, Phys. Rev. Letters 21, 1550 (1968);

D.R. Divgi and C.H. Woo, Phys. Rev. Letters 23, 1510 (1969).

[5] A. Lichnerowicz, Publ. Math. IHES 10, 293 (1961).

[6] S. Deser and J. McCarthy, Class. Quant. Grav. 7, L119 (1990). 